\title{
Gaussian Diamond Network with Adversarial Jammer
}

\author{
Soheil Mohajer and Suhas N. Diggavi \\ École Polytechnique Fédéral de Lausanne, Lausanne, Switzerland. \\ Email: $\{$ soheil.mohajer, suhas.diggavi\}@epfl.ch
}

\begin{abstract}
In this paper we consider communication from a source to a destination over a wireless network with the help of a set of authenticated relays. We focus on a special "diamond" network, where there is no direct link between the source and the destination; however the relay nodes help to establish such a communication. There is a single adversarial node which injects signals to disrupt this communication. Like the source, it can only influence the destination through the relays. We develop an approximate characterization of the reliable transmission rate in the presence of such an adversary. This is done by developing an outer bound, and demonstrating an achievable strategy that is within a constant number of bits of the outer bound, regardless of the channel values. A deterministic version of the same problem is solved exactly, yielding insights which are used in the approximate characterization.
\end{abstract}

\section{INTRODUCTION}

Wireless communication is inherently susceptible to malicious interference attempting to disrupt communications. An adversary can utilize the broadcast medium to insert disruptive signals. This problem has been well-studied for point-to-point communication, with early works in arbitrarily varying channels (AVC) [1]. This topic has not received significant attention in the context of wireless networks, where there are relay nodes to assist communication. In this paper we formulate and study coding strategies in this problem for a simple relay network.

The question of characterizing the capacity of wireless networks, even without the presence of malicious nodes, has been an open question for many decades. Recently there has been progress on this question by looking for an approximate characterization of the capacity [2]. Underlying this is an examination of the capacity of a deterministic model that focuses on the signal interaction rather than the noise [3]. Using this deterministic model, it was shown that an exact capacity characterization can be obtained in the form of an information-theoretic max-flow min-cut result; a first such result when there is both broadcast and multiple access interference in the signal interactions. This deterministic approach also gives insights that are used in obtaining the approximate characterization in noisy (Gaussian) wireless networks. In this paper we build on these ideas by first examining the impact of the adversarial node on a wireless network modeled using a linear deterministic signal interaction. In particular, we study both the deterministic and Gaussian versions of the diamond wireless network depicted in Figures 2 and 1, respectively.

The role of malicious jamming nodes in wired networks has received recent significant attention in network coded systems (see [4], [5] and references therein). However, the problem in wireless networks is quite different due to the signal interactions caused by the broadcast nature of the channel. We will utilize the fact that the disrupting signal transmitted by the adversary (see Figure 1) cause the received signals at the authenticated relays to be related to each other. We use this in order to neutralize the adversarial signal without separating it from the legitimate transmitted signal. This technique is adapted from a coding technique, termed interference neutralization, developed for the the relay-interference network in [6], [7]. The idea is that we utilize the "correlation" in the received signal at the relays to cancel part of the undesired adversarial signal. A similar idea is used in [8] for an amplify-forward relaying strategy to reduce the interference at the receiver, and assuming a sum power constraint at the relays allows to utilize a beam-forming strategy.

The main contributions of this paper are the following. We formulate the problem of adversarial jamming for a wireless (diamond) network and provide an outer bound as well as achievable strategies for this network. In particular, we show an exact characterization of reliable transmission rate for a diamond network with linear

This work was supported in part by the Swiss National Science Foundation through NCCR-MICS under grant number 51NF40-111400 and by N-CRAVE under grant number FP7 ICT-2007-215252. 
deterministic model [3]. The coding strategy for this case crucially utilizes the interference neutralization technique developed in [6]. The deterministic version of this problem is studied for some regimes of parameters in a recent work [9]. However, here we generalize the deterministic characterization for arbitrary channel parameters. More importantly, we show that the achievable strategy inspired by the deterministic analysis is within a constant number of bits of the outer bound in the Gaussian case.

We describe the problem and main results in Section II. The analysis for linear deterministic networks is given in Section III. Section IV develops the outer bound and the achievable strategy for the Gaussian case. The results present in this work lead to several natural questions on the generalizations to arbitrary networks, multiple adversaries etc. These are topics of future work.

\section{Problem Statement}

Consider the diamond network in Fig. 1, where the source wishes to send the message $W$ reliably to the destination $D$. It encodes the message and broadcasts $X^{n}$ to the relays $B_{1}$ and $B_{2}$ through the Gaussian channels. However, the relays receive interference from an adversarial node $A$, who wishes to jam the transmission by inserting noise to the system. The signal received by the relays can be written as

$$
\begin{aligned}
& y_{1}[t]=\sqrt{f_{1}} x[t]+\sqrt{g_{1}} u[t]+z_{1}[t] \\
& y_{2}[t]=\sqrt{f_{2}} x[t]+\sqrt{g_{2}} u[t]+z_{2}[t],
\end{aligned}
$$

where $x$ is the transmitted signal by the source, $u$ is the interfering signal inserted by the jammer, and $z_{1}$ and $z_{2}$ are the additive white Gaussian noise with unit variance over each channel. The relay nodes perform any (causal) processing on their received signal sequences $\left\{y_{1}[t]\right\}$ and $\left\{y_{2}[t]\right\}$ respectively, to obtain their transmitting signal sequences, $\left\{x_{1}(t)\right\}$ and $\left\{x_{2}(t)\right\}$. The received signals at the destination nodes from the Gaussian multiple access channel can be written as

$$
y[t]=\sqrt{h_{1}} x_{1}[t]+\sqrt{h_{2}} x_{2}[t]+z[t],
$$

and wishes to decode $M$ based on its received signal. We also assume equal power constraints for the source, relays, and adversarial node, that is, $\mathbb{E}\left[x^{2}\right] \leq 1, \mathbb{E}\left[x_{1}^{2}\right] \leq 1, \mathbb{E}\left[x_{2}^{2}\right] \leq 1$, and $\mathbb{E}\left[u^{2}\right] \leq 1$.

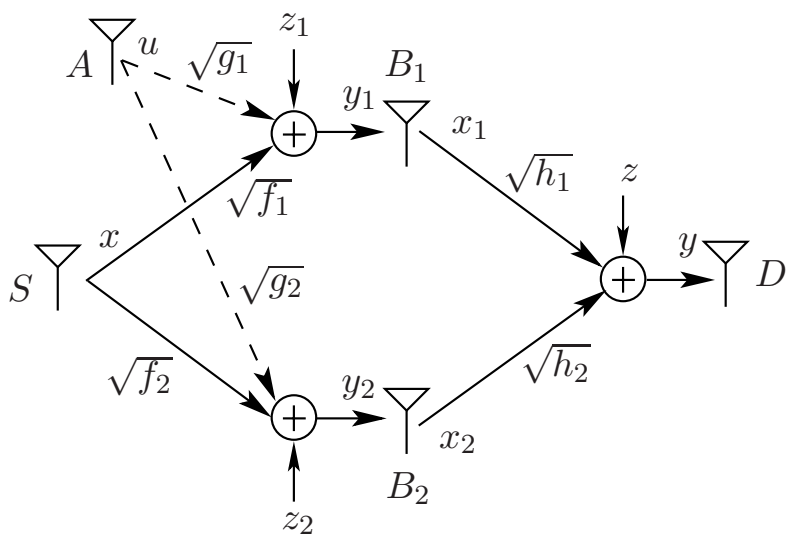

Fig. 1. Transmission model; Source node $S$ wishes to communicate to $D$, while the system is jammed by the adversarial node $A$.

The AVC problem in a point-to-point system is studied under two assumptions [1]: (a) there exists common randomness shared between the source and destination, unknown to adversary; This facilitates the use of random codebooks chosen using the common randomness; (b) if there is no such a common randomness, and a fixed codebook is used for transmission. Though we present the work for case (a), i.e., shared secret common randomness between source and the relays, these results can be easily extended to case (b).

Our main result is the (approximate) capacity from $S$ to $D$, in the presence of an adversarial jammer $A$. 
Theorem 1. The randomized capacity of the network in Figure 1 satisfies

$$
\begin{aligned}
\mathcal{C} & \leq \frac{1}{2} \log \left(1+\frac{f_{1}+f_{2}+\left(\sqrt{f_{1} g_{2}}-\sqrt{f_{2} g_{1}}\right)^{2}}{1+g_{1}+g_{2}}\right), \\
\mathcal{C} & \leq \frac{1}{2} \log \left(1+\left(\sqrt{h_{1}}+\sqrt{h_{2}}\right)^{2}\right), \\
\mathcal{C} & \leq \frac{1}{2} \log \left(1+\frac{f_{1}}{1+g_{1}}\right)+\frac{1}{2} \log \left(1+h_{2}\right), \\
\mathcal{C} & \leq \frac{1}{2} \log \left(1+\frac{f_{2}}{1+g_{2}}\right)+\frac{1}{2} \log \left(1+h_{1}\right) .
\end{aligned}
$$

Moreover, for any $\mathcal{C}$ which satisfies (1)-(4), the rate $R=\mathcal{C}-4$ is achievable.

A trivial sub-optimal scheme here is to treat the interference as independent noises, and follow the known schemes for noisy diamond relay network [3]. However, such noises are correlated since they are generated by the same jamming source. This correlation can be utilized to significantly improve the communication rate.

We first study a deterministic version of these problems by using the linear deterministic model introduced in [3]. In this model the randomness of the noise is ignored by considering the Gaussian noise's effect as limiting the precision of the received signal to a certain level, and focus on the signal interaction instead. An exact capacity characterization is obtained for the deterministic version of the problem.

The deterministic case analysis is then translated into a universally approximate characterization for the (noisy) Gaussian network. In fact, we use the insights given by analysis of the network in deterministic model for both deriving an upper bound for the capacity, as well as proposing an (approximately) optimal encoding strategy which leads to a lower bound for the capacity within a constant bit gap from the upper bound.

\section{A DETERMINISTIC APPROACH}

In this section we study the same problem in a deterministic framework introduced in [3].

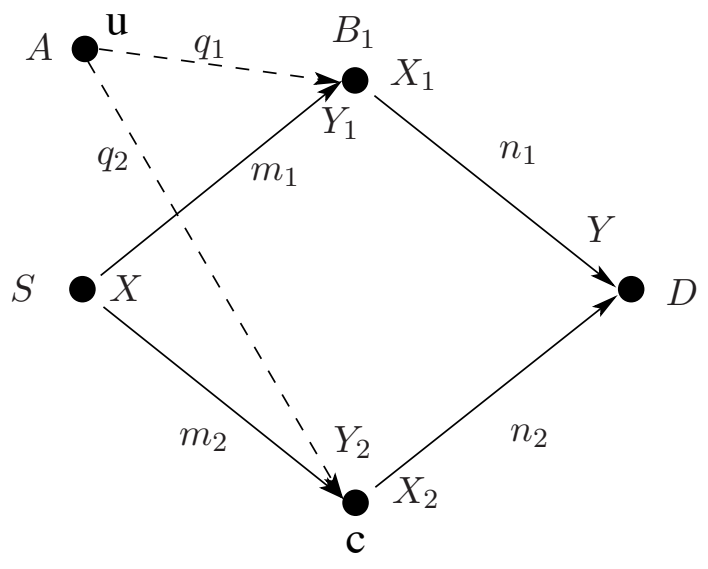

Fig. 2. The deterministic model of the problem.

The deterministic model of the problem is shown in Figure 2, where source node $S$ wishes to communicate its message to the destination node $D$ via the relay nodes $B_{1}$ and $B_{2}$. The channels from source to relays are modeled by $M_{1}$ and $M_{2}$ and from the relays to the destination are denoted by $N_{1}$ and $N_{2}$. We also use $P_{1}$ and $P_{2}$ to denote the transition matrix from the adversarial node to the relays. Similar lowercase letters are used to denote the rank of the matrices (channel gains). Therefore, the transmission over the first and second layers of the network can be respectively written as

$$
Y_{i}[t]=M_{i} X[t]+Q_{i} U[t], \quad i=1,2,
$$

and

$$
Y[t]=N_{1} X_{1}[t]+N_{2} X_{2}[t]
$$


where here $X, U, Y_{1}$ and $Y_{2}$ are vectors of length $p$ with elements form a finite field $\mathbb{F}$, and the channel matrices are powers of the lower triangular matrix $\mathbf{J}$, e.g., $M_{1}=\mathbf{J}^{p-m_{1}}$, were $\mathbf{J}$ is inspired by the linear shift deterministic model and defined as

$$
\mathbf{J}=\left(\begin{array}{ccccc}
0 & 0 & 0 & \cdots & 0 \\
1 & 0 & 0 & \cdots & 0 \\
0 & 1 & 0 & \cdots & 0 \\
\vdots & \ddots & \ddots & \ddots & \ddots \\
0 & \cdots & 0 & 1 & 0
\end{array}\right)_{p \times p}
$$

The following theorem estates the capacity characterization for this deterministic network.

Theorem 2. The capacity of the deterministic diamond network with adversarial node is given by

$$
\begin{aligned}
\mathcal{C}=\min \{ & \psi\left(m_{1}, m_{2}, q_{1}, q_{2}\right)-\max \left\{q_{1}, q_{2}\right\},\left(m_{1}-q_{1}\right)^{+}+n_{2}, \\
& \left.\left(m_{2}-q_{2}\right)^{+}+n_{1}, \max \left(n_{1}, n_{2}\right)\right\},
\end{aligned}
$$

where

$$
\Psi\left(m_{1}, m_{2}, q_{1}, q_{2}\right)= \begin{cases}\max \left\{m_{1}, m_{2}, q_{1}, q_{2}\right\} & \text { if } m_{1}+q_{2}=m_{2}+q_{1} \\ \max \left\{m_{1}+q_{2}, m_{2}+q_{1}\right\} & \text { otherwise }\end{cases}
$$

In the following we will prove this theorem.

Proof of Theorem 2:

a) The converse part: We first show that any achievable rate $R$ satisfies $R \leq \mathcal{C}$. In order to do this, we show that $R$ is upper bounded by all the four terms in the minimization in (8). Assume we use code of length $\ell$, and $W$ can be decoded from $Y^{\ell}$ with error probability $\epsilon_{\ell}$, where $\epsilon_{\ell} \rightarrow 0$ as $\ell$ grows. Therefore, using Fano's inequality, we have

$$
H\left(X^{\ell} \mid Y^{\ell}\right) \leq H\left(W \mid Y^{\ell}\right) \leq \ell \epsilon_{\ell} .
$$

We denote by $G_{X}$ and $G_{U}$ the transfer matrix from the adversarial node and the source to the relays, respectively. These matrices are defined as

$$
G_{X}=\left[\begin{array}{l}
M_{1} \\
M_{2}
\end{array}\right], \quad G_{U}=\left[\begin{array}{l}
Q_{1} \\
Q_{2}
\end{array}\right]
$$

So, the signals received by the relays can be written as

$$
\left[\begin{array}{l}
Y_{1} \\
Y_{2}
\end{array}\right]=G_{X, U}\left[\begin{array}{l}
X \\
U
\end{array}\right]
$$

where $G_{X, U}=\left[G_{X} \mid G_{U}\right]$ is the transfer matrix from $A$ and $S$ to the relay nodes. We use fold face matrices, to denote $\ell$ copy of them, as the transfer matrix applied over a codeword of length $\ell$, e.g., $\mathbf{G}_{X, U}=\mathbf{I}_{\ell} \otimes G_{X, U}$.

Note that the Markov chain $X^{\ell} \leftrightarrow\left(Y_{1}^{\ell}, Y_{2}^{\ell}\right) \leftrightarrow Y^{\ell}$ implies that the signals received at $B_{1}$ and $B_{2}$ are enough to decode the message. It is also clear that once $B_{1}$ and $B_{2}$ can decode $X$, they also know $\mathbf{G}_{U} U^{\ell}$. Hence, $H\left(X^{\ell}, \otimes \mathbf{G}_{U} U^{\ell} \mid Y_{1}^{\ell}, Y_{2}^{\ell}\right) \leq \ell \epsilon_{\ell}$. Therefore,

$$
\begin{aligned}
\ell R+H\left(\mathbf{G}_{U} U^{\ell}\right) & =H\left(X^{\ell}\right)+H\left(\mathbf{G}_{U} U^{\ell}\right)=H\left(X^{\ell}, \mathbf{G}_{U} U^{\ell}\right) \\
& \leq I\left(X^{\ell}, \mathbf{G}_{U} U^{\ell} ; Y_{1}^{\ell}, Y_{2}^{\ell}\right)+\ell \epsilon_{\ell} \\
& \leq H\left(Y_{1}^{\ell}, Y_{2}^{\ell}\right)+\ell \epsilon_{\ell}=\operatorname{rank}\left(\mathbf{G}_{X, U}\right)+\ell \epsilon_{\ell} \\
& =\ell \operatorname{rank}\left(G_{X, U}\right)+\ell \epsilon_{\ell}
\end{aligned}
$$

It is clear that the adversary can choose $U^{\ell}$ such that $H\left(\mathbf{G}_{U} U^{\ell}\right)=\ell \operatorname{rank}\left(G_{U}\right)=\ell \max \left\{q_{1}, q_{2}\right\}$. Therefore, we have $R \leq \operatorname{rank}\left(G_{X, U}\right)-\max \left\{q_{1}, q_{2}\right\}+\epsilon_{\ell}$. It only remains to show that $\operatorname{rank}\left(G_{X, U}\right)=\Psi\left(m_{1}, m_{2}, q_{1}, q_{2}\right)$, which is a known fact from linear algebra, and we skip it here to sake of brevity. Note that this bound essentially captures the maximum amount of information can be transmitted from through the cut $\Omega_{1}=\{S, A\}$. 
In order to show that $R \leq\left(m_{1}-q_{1}\right)^{+}+n_{2}$, we first recall that decodability of $W$ from $Y^{\ell}$ implies

$$
\begin{aligned}
H\left(X^{\ell}, \mathbf{Q}_{1} U^{\ell} \mid Y_{1}^{\ell}, Y^{\ell}\right) & =H\left(X^{\ell} \mid Y_{1}^{\ell}, Y^{\ell}\right)+H\left(\mathbf{Q}_{1} U^{\ell} \mid X^{\ell}, Y_{1}^{\ell}, Y^{\ell}\right) \\
& \leq H\left(W \mid Y_{1}^{\ell}, Y^{\ell}\right)+H\left(Y_{1}^{\ell}-\mathbf{M}_{1} X^{\ell} \mid X^{\ell}, Y_{1}^{\ell}, Y^{\ell}\right) \\
& \leq \ell \epsilon_{\ell} .
\end{aligned}
$$

Hence,

$$
\begin{aligned}
\ell R+H\left(\mathbf{Q}_{1} U^{\ell}\right) & =H\left(X^{\ell}\right)+H\left(\mathbf{Q}_{1} U^{\ell}\right) \\
& \stackrel{(a)}{=} H\left(X^{\ell}, \mathbf{Q}_{1} U^{\ell}\right) \\
& \leq I\left(X^{\ell}, \mathbf{Q}_{1} U^{\ell} ; Y_{1}^{\ell}, Y^{\ell}\right)+\ell \epsilon_{\ell} \\
& \left.\leq H\left(Y_{1}^{\ell}, Y^{\ell}\right)+\ell \epsilon_{\ell}\right) \\
& =H\left(Y_{1}^{\ell}\right)+H\left(Y^{\ell} \mid Y_{1}^{\ell}\right)+\ell \epsilon_{\ell} \\
& \stackrel{(b)}{\leq} H\left(Y_{1}^{\ell}\right)+H\left(\mathbf{N}_{2} X_{2}^{\ell} \mid Y_{1}^{\ell}\right)+\ell \epsilon_{\ell} \\
& \leq H\left(Y_{1}^{\ell}\right)+H\left(\mathbf{N}_{2} X_{2}^{\ell}\right)+\ell \epsilon_{\ell} \\
& \leq \ell \max \left\{m_{1}, q_{1}\right\}+\ell n_{2}+\ell \epsilon_{\ell} .
\end{aligned}
$$

where in $(a)$ we used the assumption that the adversary does not know the message, and therefore, its interfering signal is independent of $X^{\ell}$, and $(b)$ holds since $X_{1}^{\ell}$ is a function of $Y_{!}^{\ell}$. Combining (15) with the fact that the adversarial node can make $H\left(\mathbf{Q}_{1} U^{\ell}\right)$ as large as $\ell q_{1}$, gives the second bound. It is worth mentioning that this bound essentially captures the maximum flow of information through the cut $\Omega=\left\{S, A, B_{1}\right\}$. The proof of the third bound is just repeating the same argument for a symmetric situation.

Finally, in order to proof the last inequality, we consider $\Omega=\left\{S, A, B_{1}, B_{2}\right\}$. It is clear that

$$
\begin{aligned}
\ell R & \leq H\left(X^{\ell}\right) \leq I\left(X^{\ell} ; Y^{\ell}\right)+\ell \epsilon_{\ell} \\
& \leq H\left(Y^{\ell}\right)+\ell \epsilon_{\ell} \leq \ell \max \left\{n_{1}, n_{2}\right\}+\ell \epsilon_{\ell} .
\end{aligned}
$$

b)The achievability part: In the following we present an encoding scheme to achieve rates any $R \leq \mathcal{C}$. Note that is only a proof sketch, and we may skip the details due to lack of space. The idea is provide enough number of linearly independent equations about message codeword. Note that some of sub-nodes in $B_{1}$ and $B_{2}$ receive the same bit from $A$, and therefore this interfering bit can get neutralized [6] if they get forwarded and received on the same sub-node of $D$.

We split the message into two parts, which essentially leads to a network decomposition, based on the sub-levels of each node which are involved in transmitting each of the sub-messages. We first identify the maximum number of bits of the message can be neutralized using this technique. Any sub-level which is involved in such neutralization would belong the first network partition.

There are also possibly a subset of message bits received at the relays above the interference level, and therefore not corrupted with interference. These bits can be directly forwarded to the destination. This idea is illustrated in Figure 3.

We use $R^{(N)}$ and $R^{(P)}$ to denote the number of equations can be received at the destination using interference neutralization, and forwarding pure signal bits from the relays, respectively.

We denote the levels of $B_{1}$ at the receiver side by $Y_{1,1}$ (for the highest) to $Y_{1, p}$ (for the lowest), and similarly for $B_{2}$. Define

$$
\delta \triangleq \min \left\{q_{1}, q_{2}\right\}-\min \left\{\left(q_{1}-m_{1}\right)^{+},\left(q_{2}-m_{2}\right)^{+}\right\} .
$$

It is easy to show that $Y_{1,\left(p-\left(q_{1}-q_{2}\right)^{+}+\kappa\right)}$ and $Y_{2,\left(p-\left(q_{2}-q_{1}\right)^{+}+\kappa\right)}$ are corrupted by the same bit from $A$, for $\kappa=$ $0, \ldots, \delta-1$. Moreover, at least one of $Y_{1,\left(p-\left(q_{1}-q_{2}\right)^{+}+\kappa\right)}$ and $Y_{2,\left(p-\left(q_{2}-q_{1}\right)^{+}+\kappa\right)}$ receive a bit from the source. Therefore, for each $\kappa$, if $Y_{1,\left(p-\left(q_{1}-q_{2}\right)^{+}+\kappa\right)}$ and $Y_{2,\left(p-\left(q_{2}-q_{1}\right)^{+}+\kappa\right)}$ can get forwarded on the same destination subnode, the destination receives an equation about the source whose interference is neutralized. It is worth mentioning that if $m_{1}+q_{2}=m_{2}+q_{1}$, then the neutralized equations received at $D$ are zero, since the message bits would be also neutralized. On the other hand, the condition $m_{1}+q_{2} \neq m_{2}+q_{1}$ guarantees that such received legitimate bits are different. 


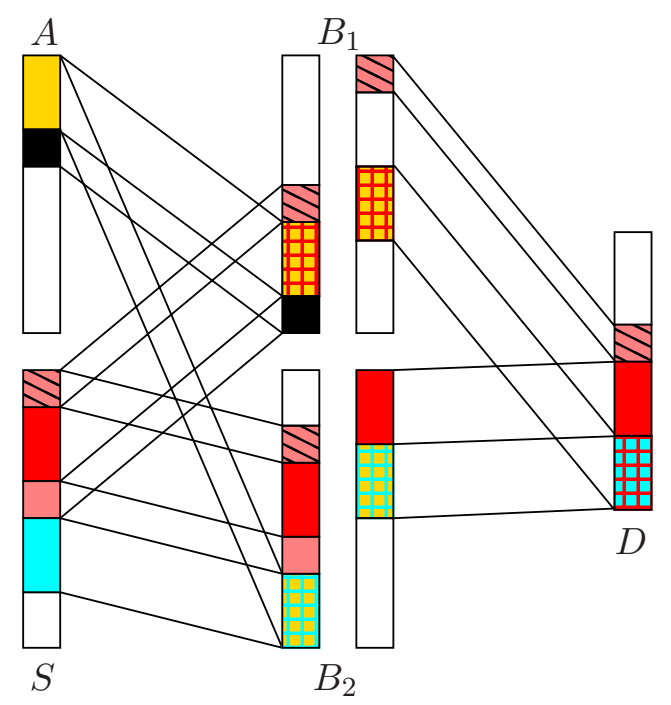

Fig. 3. Transmission strategy to provide interference neutralization.

Each bit neutralization utilizes one sub-link for $B_{1}$ to $D$, and one sub-link from $B_{2}$ to $D$. Hence, we can send up to

$$
R^{(N)} \leq \nu \triangleq \begin{cases}0 & \text { if } m_{1}+q_{2}=m_{2}+q_{1} \\ \min \left\{\delta, n_{1}, n_{2}\right\} & \text { otherwise. }\end{cases}
$$

On the other hand, nodes $B_{1}$ and $B_{2}$, respectively, receive $\left(m_{1}-q_{1}\right)^{+}$and $\left(m_{2}-q_{2}\right)^{+}$bits from the source which are above the interference level, and therefore not corrupted. These bits can be forwarded to $D$ through the remaining $\left(n_{1}-\nu\right)$ and $\left(n_{2}-\nu\right)$ links in the second layer of the network. However, these two set of bits have overlap, since they both are the upper level bits sent by $A$. Analysis of the number of non-interfered bits can be sent to the destination node, is equivalent to a study of another linear shift deterministic diamond network without adversary, where there are $\left(m_{1}-q_{1}\right)^{+}$and $\left(m_{2}-q_{2}\right)^{+}$links from $S$ to the relays, and $\left(n_{1}-\nu\right)$ and $\left(n_{2}-\nu\right)$ links from the relays to the destination. The capacity of this network is easy to compute as in [3]. We get

$$
\begin{aligned}
& R^{(P)} \leq \min \{ \max \left\{\left(m_{1}-q_{1}\right)^{+},\left(m_{2}-q_{2}\right)^{+}\right\},\left(m_{1}-q_{1}\right)^{+}+\left(n_{1}-\nu\right), \\
&\left.\left(m_{2}-q_{2}\right)^{+}+\left(n_{2}-\nu\right), \max \left\{n_{2}-\nu, n_{2}-\nu\right\}\right\} .
\end{aligned}
$$

It is easy to show that equations we receive using two method are linearly independent, and therefore any rate $R \leq R^{(N)}+R^{(P)}$ is achievable. Using some algebra and manipulations, one can show that adding the RHS's of (17) and (18), gives us the same bound claimed in the theorem.

In the following example we discuss this network decomposition idea in more details.

Example 1. Consider a diamond network with parameters $m_{1}=4, m_{2}=6, q_{1}=3, q_{2}=2, n_{1}=5$, and $n_{2}=4$. Also assume $p=7$. Theorem 2 implies that $C=5$. In the following we show how the destination can get 5 linearly independent equations about the bits transmitted by the source node. Denoting the source and interference bits by $X_{i}$ and $u_{j}$, for $i=1, \ldots, 6$, and $j=1,2,3$, we have

$$
Y_{1}=\left[\begin{array}{c}
Y_{11} \\
Y_{12} \\
Y_{13} \\
Y_{14} \\
Y_{15} \\
Y_{16} \\
Y_{17}
\end{array}\right]=\left[\begin{array}{c}
0 \\
0 \\
0 \\
X_{1} \\
X_{2}+U_{1} \\
X_{3}+U_{2} \\
X_{4}+U_{3}
\end{array}\right], \quad Y_{2}=\left[\begin{array}{c}
Y_{21} \\
Y_{22} \\
Y_{23} \\
Y_{24} \\
Y_{25} \\
Y_{26} \\
Y_{27}
\end{array}\right]=\left[\begin{array}{c}
0 \\
X_{1} \\
X_{2} \\
X_{3} \\
X_{4} \\
X_{5}+U_{1} \\
X_{6}+U_{2}
\end{array}\right] .
$$


Recall that we can neutralize up to $\nu=\min \left\{\delta, n_{1}, n_{2}\right\}=2$ bits. This will be done by forwarding $Y_{15}$ and $Y_{16}$ by $B_{1}$ and $Y_{26}$ and $Y_{27}$ by $B_{2}$ over the lowest 2 links to $D$. The destination node will receive $Y_{6}=X_{2}+X_{5}$ and $Y_{7}=X_{3}+X_{6}$ on its lowest level.

the relay node $B_{1}$ has only one bit of non-corrupted signal, $X_{1}$ and node $B_{2}$ has four of them, $X_{1}, X_{2}, X_{3}$, and $X_{4}$. In order to send these bits, $X$ send $X_{1}$ on its highest level, and $B_{2}$ forwards $X_{2}$ and $X_{3}$ on its highest levels. Note that, we cannot decode all the six bits, but obtain five linearly independently equations involving $X_{1}, \ldots, X_{6}$.

\section{The Gaussian NeTwork: Proof of Theorem 1}

The upper bound:: We imitate the same bounding techniques we used to prove Theorem 2 . We first start with the cut $\Omega=\{S, A\}$. Recall that the worst distribution the adversary can use for its signal is the Gaussian distribution [10].

$$
\begin{aligned}
\ell R & \leq I\left(y_{1}^{\ell}, y_{2}^{\ell} ; x^{\ell}\right)+\ell \epsilon_{\ell}=h\left(y_{1}^{\ell}, y_{2}^{\ell}\right)-h\left(y_{1}^{\ell}, y_{2}^{\ell} \mid x^{\ell}\right)+\ell \epsilon_{\ell} \\
& \leq \frac{\ell}{2} \log \left(\frac{1+g_{1}+g_{2}+f_{1}+f_{2}+\left(\sqrt{f_{1} g_{2}}-\sqrt{f_{2} g_{1}}\right)^{2}}{1+g_{1}+g_{2}}\right)+\ell \epsilon_{\ell} .
\end{aligned}
$$

The second bound is easy to derive.

$$
\begin{aligned}
\ell R & \leq I\left(y_{1}^{\ell}, y^{\ell} ; x^{\ell}\right)+\ell \epsilon_{\ell} \\
& =I\left(y_{1}^{\ell} ; x^{\ell}\right)+I\left(y^{\ell} ; x^{\ell} \mid y_{1}^{\ell}\right)+\ell \epsilon_{\ell} \\
& =I\left(y_{1}^{\ell} ; x^{\ell}\right)+h\left(y^{\ell} \mid y_{1}^{\ell}\right)-h\left(y^{\ell} \mid x^{\ell}, y_{1}^{\ell}\right)+\ell \epsilon_{\ell} \\
& \leq I\left(y_{1}^{\ell} ; x^{\ell}\right)+h\left(y^{\ell} \mid x_{1}^{\ell}\right)-h\left(y^{\ell} \mid x_{1}^{\ell}, x_{2}^{\ell}\right)+\ell \epsilon_{\ell} \\
& =I\left(y_{1}^{\ell} ; x^{\ell}\right)+h\left(\sqrt{h_{2}} x_{2}^{\ell}+z^{\ell} \mid x_{1}^{\ell}\right)-h\left(y^{\ell} \mid x_{1}^{\ell}, x_{2}^{\ell}\right)+\ell \epsilon_{\ell} \\
& \leq I\left(y_{1}^{\ell} ; x^{\ell}\right)+h\left(\sqrt{h_{2}} x_{2}^{\ell}+z^{\ell}\right)-h\left(y^{\ell} \mid x_{1}^{\ell}, x_{2}^{\ell}\right)+\ell \epsilon_{\ell} \\
& \leq \frac{\ell}{2} \log \left(1+\frac{f_{1}}{1+g_{1}}\right)+\frac{\ell}{2} \log \left(1+h_{2}\right)+\ell \epsilon_{\ell} .
\end{aligned}
$$

where in (20) we used the fact that $x_{1}^{\ell}$ is a function of $y_{1}^{\ell}$, and also the Markov chain $y^{\ell} \leftrightarrow\left(x_{1}^{\ell}, x_{2}^{\ell}\right) \leftrightarrow\left(x^{\ell}, y_{1}^{\ell}\right)$. The third bound can be proved by repeating the same argument for $I\left(y_{2}^{\ell}, y^{\ell} ; x^{\ell}\right)$.

In order to show the last upper bound, we can write

$$
\begin{aligned}
\ell R & \leq I\left(y^{\ell} ; x^{\ell}\right)+\ell \epsilon_{\ell} \\
& \leq I\left(y^{\ell} ; x_{1}^{\ell} x_{2}^{\ell}\right)+\ell \epsilon_{\ell} \\
& \leq \frac{\ell}{2} \log \left(1+\left(\sqrt{h_{1}}+\sqrt{h_{2}}\right)^{2}\right) .
\end{aligned}
$$

We again used the data processing inequality for the Markov chain $y^{\ell} \leftrightarrow\left(x_{1}^{\ell}, x_{2}^{\ell}\right) \leftrightarrow x^{\ell}$ in (22).

The proof for the inner bound:: The achievability scheme we propose here is induced by the strategy proposed for the deterministic model. It is based on message splitting and superposition coding. The power allocation should be performed such that the part of the message which is not corrupted by interference can be decoded at the relays. Moreover, the interfered part get forwarded to the destination such that the effective interference at the destination be small enough such that this part of the message can be decoded at the destination. In the following we only explain this idea in more details.

Without loss of generality, we assume that the relay $B_{1}$ is stronger than $B_{2}$, i.e., $\mathrm{SINR}_{1} \geq \mathrm{SINR}_{2}$, where $\operatorname{SINR}_{i}=f_{i} /\left(1+g_{i}\right)$. We first split the message $W$ into three parts, namely, $W_{c}, W_{p}, W_{n}$, with rates $R_{c}, R_{p}$, and $R_{n}$. Our encoding and decoding strategy guarantees that the common message, $W_{c}$, can be decoded at both relays, while the private sub-message, $W_{p}$, can be only decoded at $B_{1}$. However, neither of the relays can decode the neutralization sub-message, $W_{n}$, and it can be only decoded at the destination, once the interference is neutralized.

We use three random codebooks of rates $R_{c}, R_{p}$, and $R_{n}$, generated according to the Gaussian distribution with unit variance. The source maps its sub-messages to the codewords from corresponding codebooks, and obtains $\mathbf{x}_{c}$, 
$\mathbf{x}_{p}$, and $\mathbf{x}_{n}$. Then the signal transmitted by the source is formed as a super position of the three codewords, using a proper power allocation,

$$
\mathbf{x}=\sqrt{\alpha_{c}} \mathbf{x}_{c}+\sqrt{\alpha_{p}} \mathbf{x}_{p}+\sqrt{\alpha_{n}} \mathbf{x}_{n}
$$

where the power allocation coefficients satisfy

$$
\alpha_{c}+\alpha_{p}+\alpha_{n} \leq 1 .
$$

In particular, we choose $\alpha_{n}=\min \left(1,1 / \mathrm{SINR}_{1}\right), \alpha_{p}=\min \left(1,1 / \mathrm{SINR}_{2}\right)-\alpha_{n}$, and $\alpha_{c}=1-\alpha_{n}-\alpha_{p}$, which clearly satisfy the power constraint at the transmitter.

The relay nodes receive

$$
\begin{aligned}
& \mathbf{y}_{1}=\sqrt{f_{1} \alpha_{c}} \mathbf{x}_{c}+\sqrt{f_{1} \alpha_{p}} \mathbf{x}_{p}+\sqrt{f_{1} \alpha_{n}} \mathbf{x}_{n}+\sqrt{g_{1}} \mathbf{u}+\mathbf{z}_{1} \\
& \mathbf{y}_{2}=\sqrt{f_{2} \alpha_{c}} \mathbf{x}_{c}+\sqrt{f_{2} \alpha_{p}} \mathbf{x}_{p}+\sqrt{f_{2} \alpha_{n}} \mathbf{x}_{n}+\sqrt{g_{2}} \mathbf{u}+\mathbf{z}_{2} .
\end{aligned}
$$

Both nodes $B_{1}$ and $B_{2}$, first decodes $\mathbf{x}_{c}$ treating everything else as noise. Note that assuming randomized coding, this $W_{c}$ can be decoded at $B_{1}$ and $B_{2}$ as long as $R_{c}<\bar{R}_{c, 1}$ and $R_{c}<\bar{R}_{c, 2}$, where

$$
\begin{aligned}
\bar{R}_{c, 1} & \triangleq \frac{1}{2} \log \left(\frac{1+f_{1}+g_{1}}{1+f_{1}\left(\alpha_{n}+\alpha_{p}\right)+g_{1}}\right) \\
\bar{R}_{c, 2} & \triangleq \frac{1}{2} \log \left(\frac{1+f_{2}+g_{2}}{1+f_{2}\left(\alpha_{n}+\alpha_{p}\right)+g_{2}}\right) \\
& =\frac{1}{2} \log \left(\frac{1+f_{2}+g_{2}}{1+\min \left(f_{1}, g_{2}+1\right)+g_{2}}\right) \\
& >\frac{1}{2} \log \left(\frac{1+f_{2}+g_{2}}{2\left(1+g_{2}\right)}\right) \\
& =\frac{1}{2} \log \left(1+\text { SINR }_{2}\right)-\frac{1}{2} .
\end{aligned}
$$

It is easy to show that $\bar{R}_{c, 1}>\bar{R}_{c, 2}$. Therefore, any common rate satisfying

$$
R_{c}<\left(\frac{1}{2} \log \left(1+\mathrm{SINR}_{2}\right)-\frac{1}{2}\right)^{+}
$$

is achievable for the relays.

Once $\mathbf{x}_{c}$ is decoded, $B_{1}$ can cancel it from its received signal, and decode $\mathbf{x}_{p}$ treating $\mathbf{x}_{n}, \mathbf{u}$ and $\mathbf{z}_{1}$ as noise. This can be done if and only if $R_{p}<\bar{R}_{p, 1}$, where

$$
\begin{aligned}
\bar{R}_{p, 1} & \triangleq \frac{1}{2} \log \left(\frac{1+f_{1}\left(\alpha_{p}+\alpha_{n}\right)+g_{1}}{1+f_{1} \alpha_{n}+g_{1}}\right) \\
& >\frac{1}{2} \log \left(1+\text { SINR }_{1}\right)-\frac{1}{2} \log \left(1+\text { SINR }_{2}\right)-\frac{1}{2} .
\end{aligned}
$$

Therefore, any private rate satisfying

$$
R_{p}<\left(\frac{1}{2} \log \left(1+\mathrm{SINR}_{1}\right)-\frac{1}{2} \log \left(1+\mathrm{SINR}_{2}\right)-\frac{1}{2}\right)^{+}
$$

is achievable.

The operation at the relays in order to generate their transmitting signals is just to allocate proper power to their available different components. Note that the remaining (uncoded) parts of the signals will be also used for forming the transmitting signal. The relays nodes $B_{1}$ and $X_{2}$, send

$$
\begin{aligned}
& \mathbf{x}_{1}=\sqrt{\beta_{c}} \mathbf{x}_{c}+\sqrt{\beta_{p}} \mathbf{x}_{p}+\sqrt{\beta_{n}} \frac{\mathbf{y}_{1}-\sqrt{f_{1} \alpha_{c}} \mathbf{x}_{c}-\sqrt{f_{1} \alpha_{p}} \mathbf{x}_{p}}{\sqrt{f_{1} \alpha_{n}+g_{1}+1}} \\
& \mathbf{x}_{2}=\sqrt{\gamma_{c}} \mathbf{x}_{c}-\sqrt{\gamma_{n}} \frac{\mathbf{y}_{2}-\sqrt{f_{2} \alpha_{c}} \mathbf{x}_{c}}{\sqrt{f_{2} \alpha_{p}+f_{2} \alpha_{n}+g_{2}+1}},
\end{aligned}
$$


where again the power coefficients satisfy

$$
\begin{aligned}
\beta_{c}+\beta_{p}+\beta_{n} & \leq 1 \\
\gamma_{c}+\gamma_{n} & \leq 1 .
\end{aligned}
$$

Finally the decoder receivers a noisy linear combination of $\mathbf{x}_{1}$ and $\mathbf{x}_{2}$ over the multiple access channel. The signal received at the destination node can be written as

$$
\begin{aligned}
\mathbf{y}= & \sqrt{h_{1}} \mathbf{x}_{1}+\sqrt{h_{2}} \mathbf{x}_{2}+\mathbf{z} \\
= & \left(\sqrt{h_{1} \beta_{c}}+\sqrt{h_{2} \gamma_{c}}\right) \mathbf{x}_{c}+\left(\sqrt{h_{1} \beta_{p}}-\sqrt{\frac{h_{2} \gamma_{n} f_{2} \alpha_{p}}{f_{2}\left(\alpha_{p}+\alpha_{n}\right)+g_{2}+1}}\right) \mathbf{x}_{p} \\
& +\left(\sqrt{\frac{h_{1} \beta_{n} f_{1}}{f_{1} \alpha_{n}+g_{1}+1}}-\sqrt{\frac{h_{2} \gamma_{n} f_{2}}{f_{2}\left(\alpha_{p}+\alpha_{n}\right)+g_{2}+1}}\right) \sqrt{\alpha_{n}} \mathbf{x}_{n} \\
& +\left(\sqrt{\frac{h_{1} \beta_{n} g_{1}}{f_{1} \alpha_{n}+g_{1}+1}}-\sqrt{\frac{h_{2} \gamma_{n} g_{2}}{f_{2}\left(\alpha_{p}+\alpha_{n}\right)+g_{2}+1}}\right) \mathbf{u} \\
& +\left(\sqrt{\frac{h_{1} \beta_{n}}{f_{1} \alpha_{n}+g_{1}+1}} \mathbf{z}_{1}-\sqrt{\frac{h_{2} \gamma_{n}}{f_{2}\left(\alpha_{p}+\alpha_{n}\right)+g_{2}+1}} \mathbf{z}_{2}+\mathbf{z}\right) .
\end{aligned}
$$

In order to seek of simplicity, we denote the received power of $\mathbf{x}_{c}, \mathbf{x}_{p}, \mathbf{x}_{n}$ and $\mathbf{u}$ by $P_{c}, P_{p}, P_{n}$, and $P_{u}$, respectively. We also use $N$ to denote the received noise power at the destination.

We can choose the power allocation coefficients arbitrarily. In particular, we can set them such that

$$
\eta \triangleq \frac{h_{1} \beta_{n}\left(g_{1}+1\right)}{f_{1} \alpha_{n}+g_{1}+1}=\frac{h_{2} \gamma_{n}\left(g_{2}+1\right)}{f_{2}\left(\alpha_{p}+\alpha_{n}\right)+g_{2}+1} .
$$

Note that

$$
\eta<\frac{h_{1} \beta_{n}\left(g_{1}+1\right)}{g_{1}+1}=h_{1} \beta_{n} .
$$

We also have $f_{1} \alpha_{n} \leq f_{1} /$ SINR $_{1}=g_{1}+1$, which implies

$$
\eta \geq \frac{h_{1} \beta_{n}\left(g_{1}+1\right)}{2\left(g_{1}+1\right)}=h_{1} \beta_{n} / 2 .
$$

Therefore, $\eta$ is sandwiched by

$$
h_{1} \beta_{n} / 2 \leq \eta<h_{1} \beta_{n}
$$

and similarly,

$$
h_{2} \gamma_{n} / 2 \leq \eta<h_{2} \gamma_{n} .
$$

Using this new notation, we can rewrite the power of neutralization part and the jamming signal as

$$
P_{n}=\alpha_{n}\left(\sqrt{\frac{h_{1} \beta_{n} f_{1}}{f_{1} \alpha_{n}+g_{1}+1}}-\sqrt{\frac{h_{2} \gamma_{n} f_{2}}{f_{2}\left(\alpha_{p}+\alpha_{n}\right)+g_{2}+1}}\right)^{2}=\alpha_{n} \eta\left(\sqrt{\mathrm{SINR}_{1}}-\sqrt{\mathrm{SINR}_{2}}\right)^{2},
$$

and

$$
P_{u}=\left(\sqrt{\frac{h_{1} \beta_{n} g_{1}}{f_{1} \alpha_{n}+g_{1}+1}}-\sqrt{\frac{h_{2} \gamma_{n} g_{2}}{f_{2}\left(\alpha_{p}+\alpha_{n}\right)+g_{2}+1}}\right)^{2}=\eta\left(\sqrt{\frac{g_{1}}{g_{1}+1}}-\sqrt{\frac{g_{2}}{g_{2}+1}}\right)^{2} .
$$

Similarly, the total power of the noise would be

$$
N=\frac{h_{1} \beta_{n}}{f_{1} \alpha_{n}+g_{1}+1}+\frac{h_{2} \gamma_{n}}{f_{2}\left(\alpha_{p}+\alpha_{n}\right)+g_{2}+1}+1=\eta\left(\frac{1}{g_{1}+1}+\frac{1}{g_{2}+1}\right)+1 .
$$


The first task of the destination is to jointly decode $W_{c}$ and $W_{p}$, treating $\mathbf{x}_{n}$ and $\mathbf{u}$ as noise. This can be done as long as

$$
\begin{aligned}
R_{c} & \leq \frac{1}{\ell} I\left(\mathbf{y} ; \mathbf{x}_{c} \mid \mathbf{x}_{p}\right), \\
R_{p} & \leq \frac{1}{\ell} I\left(\mathbf{y} ; \mathbf{x}_{p} \mid \mathbf{x}_{c}\right), \\
R_{c}+R_{p} & \leq \frac{1}{\ell} I\left(\mathbf{y} ; \mathbf{x}_{c} \mathbf{x}_{p}\right) .
\end{aligned}
$$

The total noise and interference for decoding $\mathbf{x}_{c}$ and $\mathbf{x}_{p}$ is upper bounded by

$$
P_{n}+P_{u}+N \leq\left(\sqrt{h_{1} \beta_{n}}+\sqrt{h_{2} \gamma_{n}}\right)^{2}+1<8 \eta+1,
$$

where we used (36) and (37) in the last inequality. Therefore, the decodability conditions for $\mathbf{x}_{c}$ and $\mathbf{x}_{p}$ can be further tightened, and rewritten as

$$
\begin{aligned}
R_{p} & \leq \frac{1}{2} \log \left(\frac{h_{1}+1}{8 \eta+1}\right) \\
R_{c}+R_{p} & \leq \frac{1}{2} \log \left(\frac{h_{1}+h_{2}+1}{8 \eta+1}\right) .
\end{aligned}
$$

Combining (28), (30), (42), and (43), gives the following achievable rate.

$$
\begin{aligned}
& R_{c}+R_{p} \leq \min \left\{\frac{1}{2} \log \left(1+\mathrm{SINR}_{1}\right)-1,\right. \\
& \frac{1}{2} \log \left(1+\mathrm{SINR}_{2}\right)+\frac{1}{2} \log \left(1+h_{1}\right)-\frac{1}{2} \log (1+8 \eta)-\frac{1}{2}, \\
& \left.\frac{1}{2} \log \left(1+h_{1}+h_{2}\right)-\frac{1}{2} \log (1+8 \eta)\right\} \text {. }
\end{aligned}
$$

One $\mathbf{x}_{c}$ and $\mathbf{x}_{p}$ are decoded, the destination node can remove them and decode $\mathbf{x}_{n}$. In order to do this, it has to treat $\mathbf{u}$ as noise. Note that, $\mathbf{x}_{n}$ is decodable as long as $R_{n} \leq \bar{R}_{n}$, where

$$
\begin{aligned}
\bar{R}_{n} & \triangleq \frac{1}{2} \log \left(1+\frac{P_{n}}{P_{u}+N}\right) \\
& =\frac{1}{2} \log \left(1+\frac{\alpha_{n} \eta\left(\sqrt{\mathrm{SINR}_{1}}-\sqrt{\mathrm{SINR}_{2}}\right)^{2}}{\eta\left(\sqrt{\frac{g_{1}}{g_{1}+1}}-\sqrt{\frac{g_{2}}{g_{2}+1}}\right)^{2}+\eta\left(\frac{1}{g_{1}+1}+\frac{1}{g_{2}+1}\right)+1}\right)
\end{aligned}
$$

It is easy to show that

$$
\left(\sqrt{\frac{g_{1}}{g_{1}+1}}-\sqrt{\frac{g_{2}}{g_{2}+1}}\right)^{2}<\frac{1}{\min \left(g_{1}, g_{2}\right)+1}
$$

and

$$
\frac{1}{g_{1}+1}+\frac{1}{g_{2}+1} \leq \frac{2}{\min \left(g_{1}, g_{2}\right)+1}
$$

Therefore, we have

$$
\bar{R}_{n}>\frac{1}{2} \log \left(1+\frac{\alpha_{n} \eta\left(\sqrt{\mathrm{SINR}_{1}}-\sqrt{\mathrm{SINR}_{2}}\right)^{2}}{\eta\left(\frac{3}{\min \left(g_{1}, g_{2}\right)+1}\right)+1}\right)
$$

We can further show that

$$
\bar{R}_{n} \geq \min \left\{\frac{1}{2} \log \left(1+\frac{\alpha_{n}\left(\min \left(g_{1}, g_{2}\right)+1\right)\left(\sqrt{\mathrm{SINR}_{1}}-\sqrt{\mathrm{SINR}_{2}}\right)^{2}}{3}\right), \frac{1}{2} \log \left(1+\frac{\eta}{2}\right)\right\} .
$$


In the next step, we can prove

$$
\left(\min \left(g_{1}, g_{2}\right)+1\right)\left(\sqrt{\mathrm{SINR}_{1}}-\sqrt{\mathrm{SINR}_{2}}\right)^{2} \geq \frac{\left(\sqrt{f_{1} g_{2}}-\sqrt{f_{2} g_{1}}\right)^{2}}{3\left(g_{1}+g_{2}+1\right)} .
$$

Hence,

$$
\bar{R}_{n} \geq \min \left\{\frac{1}{2} \log \left(1+\frac{\alpha_{n}\left(\sqrt{f_{1} g_{2}}-\sqrt{f_{2} g_{1}}\right)^{2}}{9\left(g_{1}+g_{2}+1\right)}\right), \frac{1}{2} \log (1+8 \eta)-2\right\} .
$$

Summing up (44) and (51), we get the total achievable rate. It is worth mentioning that

$$
\begin{aligned}
\frac{1}{2} \log \left(1+\operatorname{SINR}_{1}\right) & +\frac{1}{2} \log \left(1+\frac{\alpha_{n}\left(\sqrt{f_{1} g_{2}}-\sqrt{f_{2} g_{1}}\right)^{2}}{9}\right) \geq \frac{1}{2} \log \left(1+\operatorname{SINR}_{1}+\frac{\left(\sqrt{f_{1} g_{2}}-\sqrt{f_{2} g_{1}}\right)^{2}}{9\left(g_{1}+g_{2}+1\right)}\right) \\
& \geq \frac{1}{2} \log \left(1+2 \operatorname{SINR}_{1}+\frac{\left(\sqrt{f_{1} g_{2}}-\sqrt{f_{2} g_{1}}\right)^{2}}{g_{1}+g_{2}+1}\right)-\frac{1}{2} \log 18 \\
& \geq \frac{1}{2} \log \left(1+\operatorname{SINR}_{1}+\operatorname{SINR}_{2}+\frac{\left(\sqrt{f_{1} g_{2}}-\sqrt{f_{2} g_{1}}\right)^{2}}{g_{1}+g_{2}+1}\right)-\frac{1}{2} \log 18 \\
& \geq \frac{1}{2} \log \left(1+\frac{f_{1}+f_{2}+\left(\sqrt{f_{1} g_{2}}-\sqrt{f_{2} g_{1}}\right)^{2}}{g_{1}+g_{2}+1}\right)-\frac{1}{2} \log 18
\end{aligned}
$$

where in the first inequality we used the fact that

$$
\left(1+\operatorname{SINR}_{1}\right) \alpha_{n}=\left(1+\operatorname{SINR}_{1}\right) \min \left(1, \frac{1}{\operatorname{SINR}_{1}}\right)=\min \left(1+\operatorname{SINR}_{1}, 1+\frac{1}{\operatorname{SINR}_{1}}\right) \geq 1 .
$$

\section{APPENDIX}

\section{A. Discussion on $\Psi\left(m_{1}, m_{2}, q_{1}, q_{2}\right)$}

Consider a block matrix with shift matrix block as

$$
G=\left[\begin{array}{ll}
\mathbf{J}^{p-m_{1}} & \mathbf{J}^{p-q_{1}} \\
\mathbf{J}^{p-m_{2}} & \mathbf{J}^{p-q_{2}}
\end{array}\right]
$$

We can, without loss of generality assume that $m_{1}=\max \left\{m_{1}, m_{2}, q_{1}, q_{2}\right\}=p$. Let $r$ denote the rank of this matrix, and our goal is to prove that $r=\Psi\left(m_{1}, m_{2}, q_{1}, q_{2}\right)$, defined in (9). Therefore, $\mathbf{G}$ can be written as a product of two full-rank matrices as

$$
G=G_{1} G_{2}=\left[\begin{array}{cc}
\mathbf{I} & \mathbf{J}^{m_{1}-q_{1}} \\
\mathbf{J}^{m_{1}-m_{2}} & \mathbf{J}^{m_{1}-q_{2}}
\end{array}\right]=\underbrace{\left[\begin{array}{cc}
\mathbf{I} & \mathbf{0} \\
\mathbf{J}^{m_{1}-m_{2}} & G_{1}^{\prime}
\end{array}\right]}_{r}\left[\begin{array}{cc}
\mathbf{I} & \mathbf{J}^{m_{1}-q_{1}} \\
\mathbf{0} & G_{2}^{\prime}
\end{array}\right]\} r
$$

where $\mathbf{I}$ is the identity matrix of size $p \times p$ and $\mathbf{0}$ is the zero matrix of size $p \times(r-p)$. Moreover, $G_{1}$ and $G_{2}$ are full-rank matrices which satisfy

$$
\mathbf{J}^{2 m_{1}-m_{2}-q_{1}}+G_{1} G_{2}=\mathbf{J}^{m_{1}-q_{2}} .
$$

It is clear that the fact that $G_{1}$ is full-rank, implies $G_{1}^{\prime}$ is also full-rank, and moreover, the first $p$ columns and the last $(r-p)$ columns of $\mathbf{G}_{1}$ are linearly independent. Therefore, $r=\operatorname{rank}\left(G_{1}\right)=p+\operatorname{rank}\left(G_{1}^{\prime}\right)$.

Note that if $m_{1}+q_{2}=m_{2}+q_{2}$, then $2 m_{1}-m_{2}-q_{1}=m_{1}-q_{2}$, and $G_{1}=G_{2}=\mathbf{0}$. Recall that $G_{1}$ and $G_{2}$ are full-rank. So, they should have zero columns, and hence $r=m_{1}$, in this case.

On the other hand, if $m_{1}+q_{2} \neq m_{2}+q_{2}$, then $G_{1}^{\prime} G_{2}^{\prime}=\mathbf{J}^{m_{1}-q_{2}}-\mathbf{J}^{2 m_{1}-m_{2}-q_{1}}$, and

$$
\operatorname{rank}\left(G_{1}^{\prime} G_{2}^{\prime}\right)=\max \left\{p-\left(m_{1}-q_{2}\right), m_{1}-\left(2 m_{1}-m_{2}-q_{1}\right)\right\} .
$$

Since $G_{1}^{\prime}$ and $G_{2}^{\prime}$ are also full-rank, each of them is of the same rank. Therefore,

$$
r=p+\operatorname{rank}\left(G_{1}^{\prime}\right)=m_{1}+\max \left\{q_{2}, m_{2}+q_{1}-m_{1}\right\}=\max \left\{m_{1}+q_{2}, m_{2}+q_{1}\right\} .
$$




\section{B. Evaluation of $R^{(N)}+R^{(P)}$}

Note that combining (17) and (18), we conclude any rate satisfying

$$
\begin{gathered}
R \leq \nu+\min \left\{\max \left\{\left(m_{1}-q_{1}\right)^{+},\left(m_{2}-q_{2}\right)^{+}\right\},\left(m_{1}-q_{1}\right)^{+}+\left(n_{1}-\nu\right),\right. \\
\left.\left(m_{2}-q_{2}\right)^{+}+\left(n_{2}-\nu\right), \max \left\{n_{2}-\nu, n_{2}-\nu\right\}\right\}
\end{gathered}
$$

is achievable.

First assume $m_{1}+q_{2}=m_{2}+q_{2}$. In this case, from (17) we have $\nu=0$. Therefore, (53) can be written as

$$
R \leq \min \left\{\left(m_{1}-q_{1}\right)^{+},\left(m_{1}-q_{1}\right)^{+}+n_{1},\left(m_{2}-q_{2}\right)^{+}+n_{2}, \max \left\{n_{2}, n_{2}\right\}\right\} .
$$

It remains to show that $\left(m_{1}-q_{1}\right)^{+}=\max \left\{m_{1}, m_{2}, q_{1}, q_{2}\right\}-\max \left\{q_{1}, q_{2}\right\}$, which is straight-forward, since we assumed $m_{1}+q_{2}=m_{2}+q_{1}$.

In the second case, where $m_{1}+q_{2} \neq m_{2}+q_{2}$, we have $\nu=\min \left\{n_{1}, n_{2}, \delta\right\}$. Therefore, (53) can be written as

$$
\begin{aligned}
R & \leq \min \left\{\max \left\{\left(m_{1}-q_{1}\right)^{+},\left(m_{2}-q_{2}\right)^{+}\right\}+\nu,\left(m_{1}-q_{1}\right)^{+}+n_{1},\left(m_{2}-q_{2}\right)^{+}+n_{2}, \max \left\{n_{2}, n_{2}\right\}\right\} \\
& =\min \left\{\max \left\{\left(m_{1}-q_{1}\right)^{+},\left(m_{2}-q_{2}\right)^{+}\right\}+\min \left\{\delta, n_{1}, n_{2}\right\},\left(m_{1}-q_{1}\right)^{+}+n_{1},\left(m_{2}-q_{2}\right)^{+}+n_{2}, \max \left\{n_{2}, n_{2}\right\}\right\} \\
& =\min \left\{\max \left\{\left(m_{1}-q_{1}\right)^{+},\left(m_{2}-q_{2}\right)^{+}\right\}+\delta,\left(m_{1}-q_{1}\right)^{+}+n_{1},\left(m_{2}-q_{2}\right)^{+}+n_{2}, \max \left\{n_{2}, n_{2}\right\}\right\}
\end{aligned}
$$

where the last equality holds, since if $\delta=n_{1}$ then the first expression is always greater than or equal to the second term, and does not appear in the minimization result. Similarly if $\delta=n_{2}$. Now, without loss of generality, we can assume that $m_{1}+q_{2}>m_{2}+q_{1}$, and conclude $\left(m_{1}-q_{1}\right)^{+} \geq\left(m_{2}-q_{2}\right)^{+}$. Hence,

$$
\begin{aligned}
\delta+\max \left\{\left(m_{1}-q_{1}\right)^{+},\left(m_{2}-q_{2}\right)^{+}\right\}= & \min \left\{q_{1}, q_{2}\right\}-\min \left\{\left(q_{1}-m_{1}\right)^{+},\left(q_{2}-m_{2}\right)^{+}\right\} \\
& +\max \left\{\left(m_{1}-q_{1}\right)^{+},\left(m_{2}-q_{2}\right)^{+}\right\} \\
= & \left(q_{1}+q_{2}-\max \left\{q_{1}, q_{2}\right\}\right)-\left(q_{1}-m_{1}\right)^{+}+\left(m_{1}-q_{1}\right)^{+} \\
= & \left(q_{1}+q_{2}-\max \left\{q_{1}, q_{2}\right\}\right)-\left(\max \left\{q_{1}, m_{1}\right\}-m_{1}\right)+\left(\max \left\{q_{1}, m_{1}\right\}-q_{1}\right) \\
= & q_{1}+q_{2}-\max \left\{q_{1}, q_{2}\right\}+m_{1}-q_{1} \\
= & m_{1}+q_{2}-\max \left\{q_{1}, q_{2}\right\} \\
= & \max \left\{m_{1}+q_{2}, m_{2}, q_{1}\right\}-\max \left\{q_{1}, q_{2}\right\}
\end{aligned}
$$

Replacing this in the (55) in (54) gives us the desired result.

\section{REFERENCES}

[1] R. Ahlswede, "Elimination of correlation in random codes for arbitrarily varying channels," Z. Wahrscheinlichkeitstheorie Verw. Gebiete, vol. 44, pp. 159-175, 1978 .

[2] A. S. Avestimehr, S. N. Diggavi, and D. N. C. Tse, "Wireless network information flow: A deterministic approach," 2009, submitted to IEEE Trans. Inform. Theory, Available from http://arxiv.org/pdf/0906.5394.

[3] A. Avestimehr, S. Diggavi, and D. Tse, "A deterministic approach to wireless relay networks," in Proceedings of Allerton Conference on Communication, Control, and Computing, Illinois, USA, Sept. 2007.

[4] R. Koetter and F. Kschischang, "Coding for errors and erasures in random network coding," IEEE Trans. Inform. Theory, vol. 54, pp. 3579-3591, Aug. 2008.

[5] S. Jaggi, M. Langberg, S. Katti, T. Ho, D. Katabi, M. Médard, and M. Effros, "Resilient network coding in the presence of byzantine adversaries," IEEE Trans. Inform. Theory, vol. 54, pp. 2596-2603, June 2008.

[6] S. Mohajer, S. N. Diggavi, C. Fragouli, and D. Tse, "Transmission techniques for relay-interference networks," in Proceedings of Allerton Conference on Communication, Control, and Computing, Illinois, USA, Sept. 2008.

[7] S. Mohajer, S. N. Diggavi, , and D. Tse, "Approximate capacity of a class of gaussian relay-interference networks," in Proceedings of IEEE Int. Symp. Inform. Theory, Seoul, Korea, July 2009.

[8] K. Gomadam and S. A. Jafar, "The effect of noise correlation in amplifyand-forward relay networks," IEEE Trans. Inform. Theory, vol. 55, no. 2, pp. 731-745, Feb. 2009.

[9] S. M. H. T. Yazdi and M. R. Aref, "The capacity of a class of linear deterministic networks," 2010, available from http://arxiv.org/pdf/1001.2164.

[10] S. Diggavi and T. Cover, "The worst additive noise under a covariance constraint," IEEE Trans. Inform. Theory, vol. 47, pp. 3072-3081, July 2001. 